\title{
Graphene Plasmons in Triangular Wedges and
}

\section{Grooves}

\author{
P. A. D. Gonçalves, ${ }^{*}, *$ E. J. C. Dias, ${ }^{\text {II }}$ Sanshui Xiao, ${ }^{\dagger}, \$$ M. I. Vasilevskiy, ${ }^{\mathbb{I}}{ }^{\mathrm{N}} \mathrm{N}$. \\ Asger Mortensen, ${ }^{\dagger} \ddagger$ and N. M. R. Peres $*, \mathbb{I}$ \\ Department of Photonics Engineering, Technical University of Denmark, DK-2800 Kgs. Lyngby, \\ Denmark, Center for Nanostructured Graphene, Technical University of Denmark, DK-2800 Kgs. \\ Lyngby, Denmark, and Department of Physics and Center of Physics, University of Minho, \\ PT-4710-057, Braga, Portugal \\ E-mail: padgo@fotonik.dtu.dk; peres@fisica.uminho.pt
}

\begin{abstract}
The ability to effectively guide electromagnetic radiation below the diffraction limit is of the utmost importance in the prospect of all-optical plasmonic circuitry. Here, we propose an alternative solution to conventional metal-based plasmonics by exploiting the deep subwavelength confinement and tunability of graphene plasmons guided along the apex of a graphene-covered dielectric wedge or groove. In particular, we present a quasi-analytic model to describe the plasmonic eigenmodes in such a system, including the complete determination of their spectrum and corresponding induced potential and electric field distributions. We have found that the dispersion of wedge/groove graphene plasmons follows the same functional dependence as their flat-graphene plasmons counterparts, but now scaled by a (purely) geometric

\footnotetext{
${ }^{*}$ To whom correspondence should be addressed

†Department of Photonics Engineering, Technical University of Denmark, DK-2800 Kgs. Lyngby, Denmark Center for Nanostructured Graphene, Technical University of Denmark, DK-2800 Kgs. Lyngby, Denmark

${ }^{\mathbb{T}}$ Department of Physics and Center of Physics, University of Minho, PT-4710-057, Braga, Portugal
} 
factor in which all the information about the system's geometry is contained. We believe our results pave the way for the development of novel custom-tailored photonic devices for subwavelength waveguiding and localization of light based on recently discovered 2D materials.

Over the last couple of decades we have been witnessing a steady, exponential growth in the amount of information produced on a daily basis. In today's "information age", huge amounts of data must be processed, stored, and delivered around the world. While the data-processing part is still primarily carried by electronics, the routing of large volumes of information is handled with photonic technologies since only these can meet the requirements in terms of highspeed, density and bandwidth. One of the greatest ambitions of modern nanophotonics 1 is to bridge the gap between electronic and photonic components, and ultimately to replace electronic circuits and processing units by their photonic-based counterparts. Current scalable photonicbased communications, however, still could not surpass the threshold towards miniaturization posed by the diffraction limit. In this regard, a great deal of hope $\mathrm{e}^{\sqrt{2}}$ has been deposited in the sub-discipline of photonics known as plasmonics, $\frac{3 \sqrt{4}}{4}$ which exploits the ability of surface plasmonpolaritons (SPPs) — collective oscillations of the free-electrons at metal/dielectric interfaces - to localize light into subwavelength dimensions. ${ }^{5-8}$ Although the pursuit of plasmonic devices suitable for mass-production is still going on, plasmonics has already achieved some milestones, for instance, subwavelength plasmonic circuitry including waveguides, interferometers and resonators, ${ }^{9-12}$ nanolasers, ${ }^{13-16}$ quantum optics with or mediated by SPPs,,$\frac{17-21}{21}$ label-free and single molecule biochemical sensing, ${ }^{22-\sqrt{26}}$ high-resolution nanoscopy, $27 / 28$ and even cancer theranostics. 29.31

A key component in any plasmonic circuit would be an element to transfer and guide the electromagnetic (EM) fields from point $A$ to point $B$. Typical SPP-guiding structures ${ }^{32}$ consist in small metallic stripes on a dielectric substrate, ${ }^{3 / 32}$ chains of plasmonic nanoparticles,, $33-35$ metal/dielectric/metal slabs, $\stackrel{32}{32}$ or V-shaped grooves carved into a metallic substrate,,$[36-38$ just to name a few. Among these, the latter are believed to be appealing candidates for subwavelength waveguiding of light since they support SPP modes coined as channel plasmon-polaritons (CPPs), 
which have been shown to deliver localized EM fields with relatively long propagation lengths, 12 [36/39 ability to work at telecommunication wavelengths, $\underline{10 / 40 \mid 41}$ and feasibility to steer the EM field along bends. ${ }^{10[42}$ The earliest reference on CPPs can be found in a theoretical investigation carried out by Dobrzynski and Maradudin, having obtained analytic expressions in the electrostatic limit for an infinitely sharp wedge. ${ }^{43}$ Many subsequent works then followed in similar wedge configurations with a rounded edge. ${ }^{44}$ During the past decade-owing to the rapid progresses in nanofabrication and computational tools - a renewed interest has emerged on CPPs guided along triangular grooves sculpted in metal substrates, leading to a plethora of theoretical and experimental studies. $36 \mid 38 / 40,48-51$.

In recent years, graphene $\sqrt{52 \mid 53}$ —an atomically-thin $s p^{2}$-hybridized carbon allotrope in which the atoms sit at the vertexes of a honeycomb lattice-has come to the light as a novel plasmonic material. ${ }^{54}$ Graphene is classified as a two-dimensional (2D) semi-metal whose charge-carriers exhibit a linear dispersion. ${ }^{52[53}$ When doped, graphene also sustains plasmon-polaritons that inherit the extraordinary optoelectronic properties of this material. In particular, gate-tunable graphene surface plasmons (GSPs) have been shown to deliver highly confined EM fields into deep subwavelength regions, large field-enhancements, strong light-matter interactions, and carry the prospect of low-loss plasmonics. $\frac{54}{59}$ In addition, the ability to easily control the carrier-density in graphene, e.g. by electrostatic gating and/or chemical means, constitutes a major advantage of GSPs over conventional metal-based plasmonics. Popular configurations to realize GSPs involve the nanostructuring of an otherwise continuous graphene sheet into graphene ribbons,, $26-60-63$ disks, $64+68$ rings,, $64[65]$ and graphene anti-dots (either as individual structures or in periodic arrays). $67[69] 70$

Here, we propose a different approach to deliver strongly localized GSPs which does not involve any nanopatterning done on the graphene layer; it simply consists in depositing graphene onto a V-shaped wedge or groove previously sculpted in the receiving substrate (a different, but related configuration was the subject of a previous numerical study ${ }^{71}$ ). This can be done by employing the same techniques used to fabricate metallic grooves, $\frac{36}{36}$ followed by the graphene deposition or even direct-growth on a pre-configured copper substrate. ${ }^{72}$ Other possibilities include 
folding a graphene layer or by exploring the formation of wrinkles (either naturally occuring $73-75$ or deliberately formed ${ }^{72}$ ). In this way, one departures from customary flat-graphene geometries and effectively produces a 1D channel which not only confines light in the vertical direction that bisects the channel, but is also capable of producing lateral confinement of EM radiation.

In this work, we present a quasi-analytic method to derive the dispersion relation and corresponding spatial distributions of the potential and electric fields akin to GSPs guided along a V-shaped channel. We shall consider both the wedge and groove geometries—see Fig. 1. In what
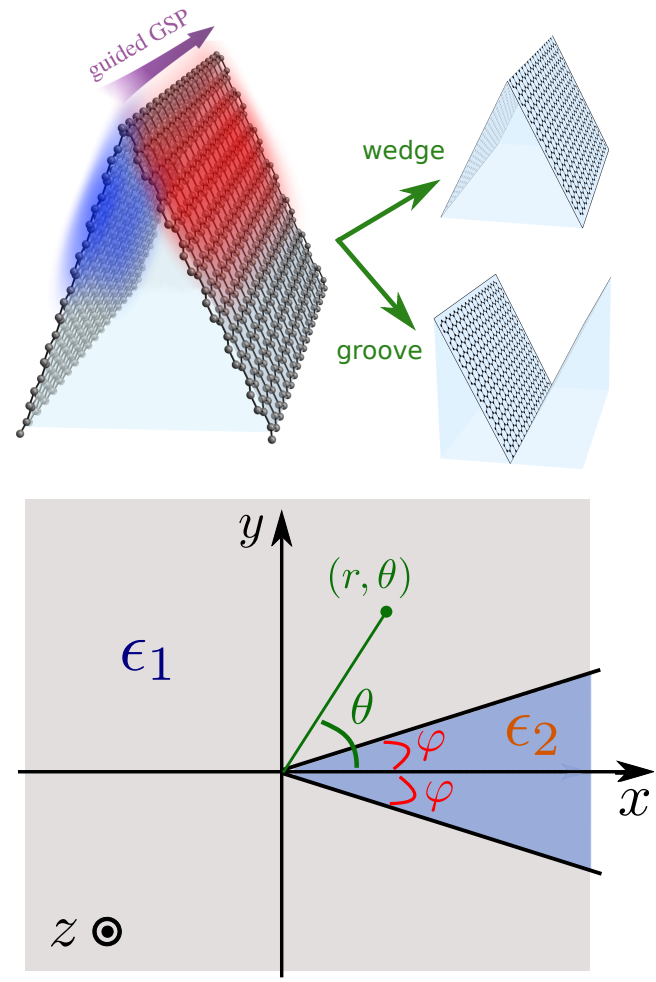

Figure 1: Upper panel: pictorial representation of a guided GSP mode along a dielectric wedge; we denote the system a wedge (groove) whenever the medium filling the $2 \varphi$-open region possesses a higher (lower) value for the relative permittivity. Lower panel: coordinate system for a wedge or groove interface-with an opening angle of $2 \varphi$ - between two dielectric media characterized by relative permittivities $\varepsilon_{2}$ (for $-\varphi \leq \theta \leq \varphi$ ) and $\varepsilon_{1}$ (for $\varphi \leq \theta \leq 2 \pi-\varphi$ ).

follows we work within the electrostatic limit, which turns out to be a very good approximation for GSPs owing to the large wavevectors carried by plasmons in graphene (and thus retardation becomes unimportant). Interestingly, we find that for a fixed wedge/groove angle the corresponding wedge/groove graphene plasmon (WGP/GGP) dispersions follow a universal scaling law that 
depends purely on the system's geometry. Thus, by performing the calculations for a given angle, $\varphi$, one immediately gains complete knowledge of the WGPs/GGPs' wavevectors for every frequency - in other words, all the modes and corresponding dispersion relations are obtained at once. This constitutes an enormous advantage in terms of computational resources and time when gauged against full-wave numerical simulations. From a device-engineering perspective this scaling property should also significantly ease design of waveguides for given applications. Finally, in further support of the accuracy of our quasi-analytic technique, we have also performed rigorous electrodynamic simulations with the aid of a commercially available (Comsol MultiPhysics) finite-element method (FEM), to which we have obtained a remarkable agreement.

We demonstrate that by using graphene-covered triangular wedges or grooves one can harness the unique properties of GSPs to create novel 1D subwavelength plasmonic waveguides that can squeeze light into deep subwavelength regimes. This becomes particularly relevant at $\mathrm{THz}$ and mid-IR frequencies since traditional metal-based plasmonics perform poorly in this spectral range (resembling freely propagating light). ${ }^{3}$ Furthermore, we believe that this work can set the stage for future investigations of graphene plasmons in $1 \mathrm{D}$ channels, with potentially relevant applications ranging from plasmonic circuitry and waveguiding to biochemical sensing with WGPs/GGPs or their integration with microfluidics on a chip.

\section{Results and Discussion}

We consider an idealized geometry in which a graphene monolayer is sandwiched between a triangular dielectric wedge (or groove) with relative permittivity $\varepsilon_{2}$ and a capping dielectric material with relative permittivity $\varepsilon_{1}$, as depicted in Fig. 1. As it will become apparent later, our model is completely general irrespective of the specific values for the dielectric constants of the cladding insulators. However, in order to cope with the standard nomenclature, we shall refer to a wedge whenever $\varepsilon_{2}>\varepsilon_{1}$ and vice-versa to denote a groove. In addition, albeit here we are primarily interested in graphene, the theory outlined below can be applied to any 2D layer deposited onto the 
triangular shape, be it a 2D electron gas or a doped 2D transition metal dichalcogenide (TMDC), etc.

Before proceeding to the description of our quasi-analytic method, we first bring to the reader's attention that one can treat the cases of even and odd symmetry in the potential (or induced charges) — with respect to the line bisecting the triangular cross-section-separately, as this makes the problem more amenable to handle. In particular, for the case of even symmetry (i.e., when the induced charges are symmetric in the graphene half-planes which constitute the Vshape), we have found that these even-symmetry modes are not highly confined near the apex of the wedge/groove, with their dispersion being virtually the same as for GSPs in a flat, planar dielectric/graphene/dielectric interface (cf. SI). Conversely, as it will become clear ahead, the corresponding odd WGPs/GGPs modes exhibit strong field confinement near the apex of the wedge/groove, and therefore we shall limit our discussion solely to the odd-symmetry case hereafter. Owing to the high localization of the field near the apex, we note that although we assume (for simplicity) an infinitely long V-shape, the theory developed here remains adequate in the description of V-structures of finite height/depth as long as their size is larger than the region spanned by the field distribution along the axis of symmetry.

The extremely large wavevectors (when compared with light's free-space wavevector, $k_{0}=$ $\omega / c)$ attained by graphene plasmons allow us to treat plasmonic excitations in graphene within the electrostatic limit with high accuracy. In this regime, the induced electric potential akin to GSPs must satisfy Poisson's equation, which in cylindric coordinates reads

$$
\left[\frac{\partial^{2}}{\partial r^{2}}+\frac{1}{r} \frac{\partial}{\partial r}+\frac{1}{\partial r^{2}} \frac{\partial^{2}}{\partial \theta^{2}}-q^{2}\right] \Phi(r, \theta)=-\frac{\rho(r, \theta)}{\varepsilon_{0}}
$$

where we have written the scalar potential as $\Phi(\mathbf{r})=\Phi(r, \theta) e^{i q z}$, owing to the system's translational invariance along the $z$-axis (an implicit time-dependence of the form $e^{-i \omega t}$ is assumed). This effectively reduces our initial $3 \mathrm{D}$ problem into a $2 \mathrm{D}$ one, and will allow us to parameterize the dispersion relation of the guided GSPs in terms of the propagation constant $q$, i.e. $\omega \equiv \omega(q)$. Hence, 
the solution of Eq. (??) renders the WGPs/GGPs modes which propagate along the longitudinal direction. Formally, the solution of this equation in the medium $j=1,2$ can be written as

$$
\Phi(r, \theta)=\frac{i \sigma(\omega)}{\omega} \int_{0}^{\infty} d r^{\prime} G_{j}\left(r, \theta ; r^{\prime}, \varphi\right)\left[\frac{\partial^{2}}{\partial r^{\prime 2}}-q^{2}\right] \Phi\left(r^{\prime}, \varphi\right)
$$

where $\sigma(\omega)$ is the dynamical conductivity of graphene, and $G_{j}\left(r, \theta ; r^{\prime}, \varphi\right)$ is the Green's function associated with Eq. (??) in that medium; the latter is defined explicitly in the SI. Moreover, when writing the preceding equation, we have expressed the carrier-density as $\rho(r, \theta)=$ -en $(r) \delta(\theta-\varphi) / r$, where the 2D particle density, $n(r)$, was written in terms of the electrostatic potential by combining the continuity equation together with Ohm's law (cf. SI). We further remark that we only need to solve for the potential in, say, the upper-half space $(0 \leq \theta \leq \pi)$, since we are looking for solutions in which the potential is odd with respect to the symmetry axis. It is clear from Eq. (??) that the potential in the whole space can only be derived once the potential at the graphene sheet (i.e., $\theta=\varphi$ ) is determined. To that end, we set $\theta=\varphi$ and then employ an orthogonal polynomials expansion technique $\frac{54 / 76 / 77}{\sqrt{6}}$ to transform the above integro-differential equation for the potential at the graphene, $\phi(r) \equiv \Phi(r, \varphi)$, into a standard linear algebra eigenproblem. This is done by expanding the electrostatic potential evaluated at the graphene layer as $\phi(r)=\sum_{n} c_{n} L_{n}^{(0)}(q r) e^{-q r / 2}$, with $L_{n}^{(0)}$ denoting the generalized Laguerre polynomials, $\stackrel{78}{ }$ and where the $c_{n}$ 's are the entries of the eigenvectors defined by the following eigensystem (obtained by exploiting the appropriate orthogonality relations $\frac{78}{}$ ):

$$
\frac{i \omega}{q \sigma(\omega)} c_{m}=\sum_{n=0}^{\infty} U_{m n} c_{n}
$$

where the matrix elements $U_{m n}$ read

$$
\begin{aligned}
U_{m n}= & \int_{0}^{\infty} \int_{0}^{\infty} d x d y G(x, \varphi ; y, \varphi) e^{-\frac{x+y}{2}} L_{m}^{(0)}(x) \\
& \times\left[\frac{3}{4} L_{n}^{(0)}(y)-L_{n-2}^{(2)}(y)-L_{n-1}^{(1)}(y)\right] .
\end{aligned}
$$


We note that the double integration over the dimensionless variables $x=q r$ and $y=q r^{\prime}$ can be performed analytically, thereby making the computation of the matrix elements extremely fast. Notice that we have dropped the index $j$ in the Green's function because the boundary condition at $\theta=\varphi$ enforces that $G_{1}(x, \varphi ; y, \varphi)=G_{2}(x, \varphi ; y, \varphi)$, and therefore one can choose either Green's function arbitrarily without any loss of generality.

The eigenvalue equation (??) can be solved numerically using standard linear-algebra routines. Once we find the corresponding eigenvalues $\tilde{\lambda}_{n}$ (whose number matches the length of the vector $\vec{c}$, i.e. $N+1$, where $N$ truncates the expansion for $\phi(r)$, and convergence was checked empiricallycf. SI), the spectrum of graphene plasmons traveling along the triangular wedge/groove straightforwardly follows from

$$
\frac{i \omega}{q \sigma(\omega)}=\tilde{\lambda}_{n}
$$

where, for a given opening angle $2 \varphi$, Eq. (??) returns a discrete set of WGPs/GGPs modes. We stress that all the momentum and frequency dependence stems from the LHS of the previous equation; hence, the eigenvalues $\tilde{\lambda}_{n} \equiv \tilde{\lambda}_{n}(\varphi)$ carry a purely geometric meaning since they depend uniquely on the configuration of the system (opening angle and material parameters). In particular, using graphene's Drude-like conductivity with negligible damping, $\underline{54}$ one obtains a "universal scaling law" for the dispersion relation of wedge/groove graphene plasmons,

$$
\Omega(q)=\Omega_{\text {flat }}(q) \frac{2}{\pi} \sqrt{\lambda_{n}}
$$

where the relation $\tilde{\lambda}_{n}=\frac{4}{\pi^{2} \varepsilon_{0}\left(\varepsilon_{1}+\varepsilon_{2}\right)} \lambda_{n}$ has been used, and stems from factorizing a constant proportionality factor entering in the Green's function (see the text after Eq. (S33) in the SI). The above equation gives the energy of the guided graphene plasmon modes parameterized by the propagation constant along the apex of the wedge. Here, $\Omega_{\text {flat }}(q)=\sqrt{\frac{4 \alpha \hbar c}{\varepsilon_{1}+\varepsilon_{2}} E_{F}} q$ is simply the dispersion relation followed by GSPs in flat graphene ${ }^{54}$ sandwiched between two dielectrics with $\varepsilon_{1}$ and $\varepsilon_{2}$ (where $\alpha \simeq 1 / 137$ denotes the fine-structure constant). Notice that once we have determined $\lambda_{n}$, we possess complete knowledge of the WGPs/GGPs spectrum-for any point in the entire $(q, \omega)$ - 
space - all of this with only one computation. In fact, we can even plot the dispersion of distinct 2D materials that support SPPs modes from a single computation of $\lambda_{n}$, since the latter does not depend on the 2D conductivity that characterizes the particular 2D material [recall Eq. (??)]. It is instructive to note that, in general, the spectrum of WGPs/GGPs contains a discrete set of even and odd modes (although here we describe only modes with odd-symmetry for the reason stated above in the text), in a similar way to SPPs supported at metallic wedges/grooves. $\frac{36}{36}$ This is a consequence of the lateral confinement near the tip of the wedge (or the bottom of the groove), and bears some resemblance to finding the electronic eigenstates of a particle in a quantum wire. ${ }^{79}$

In Fig. 2 we have plotted the dispersion relation of graphene plasmons guided along the edge of triangular wedges and grooves with different opening angles, $2 \varphi$ (indicated in the insets), which, as we have already anticipated, consists in a discrete set of well-defined modes with increasing energy. The figure plainly shows that the spectrum of both WGPs and GGPs strongly depend on the angles of the triangular opening, with smaller angles rendering correspondingly larger plasmon wavevectors for the same frequency, which in turn is an indication of stronger field confinement near the apex of the V-shape. Another striking feature visible in the figure is the outstanding agreement between the quasi-analytic theory detailed above and the electrodynamic simulations using the FEM technique (see SI for details on the simulations). This constitutes further evidence of the ability of our quasi-analytic method to render accurate results, while also providing a deeper fundamental understanding of the scaling properties. Also from the inspection of Fig. 2, a strong contrast can be perceived between the dispersion curves akin to WGPs and GGPs, demonstrating the superiority of the former in squeezing light below the diffraction limit as they attain larger wavevectors for the same angle of the structure. We further note that one can transform a wedge into a groove and vice-versa either by swapping the values of $\varepsilon$ or by applying the angular transformation $\varphi \rightarrow \pi-\varphi$ (this essentially interchanges the Green's functions $G_{1}$ and $G_{2}$ ). For the sake of completeness, in Fig. $2 \mathrm{f}$ panel we have portrayed the spectrum of GSPs guided along a triangular apex embedded in a homogeneous dielectric medium with the same average relative permittivity as its wedge and groove counterparts. It can be observed that-for the same angle-each of the 

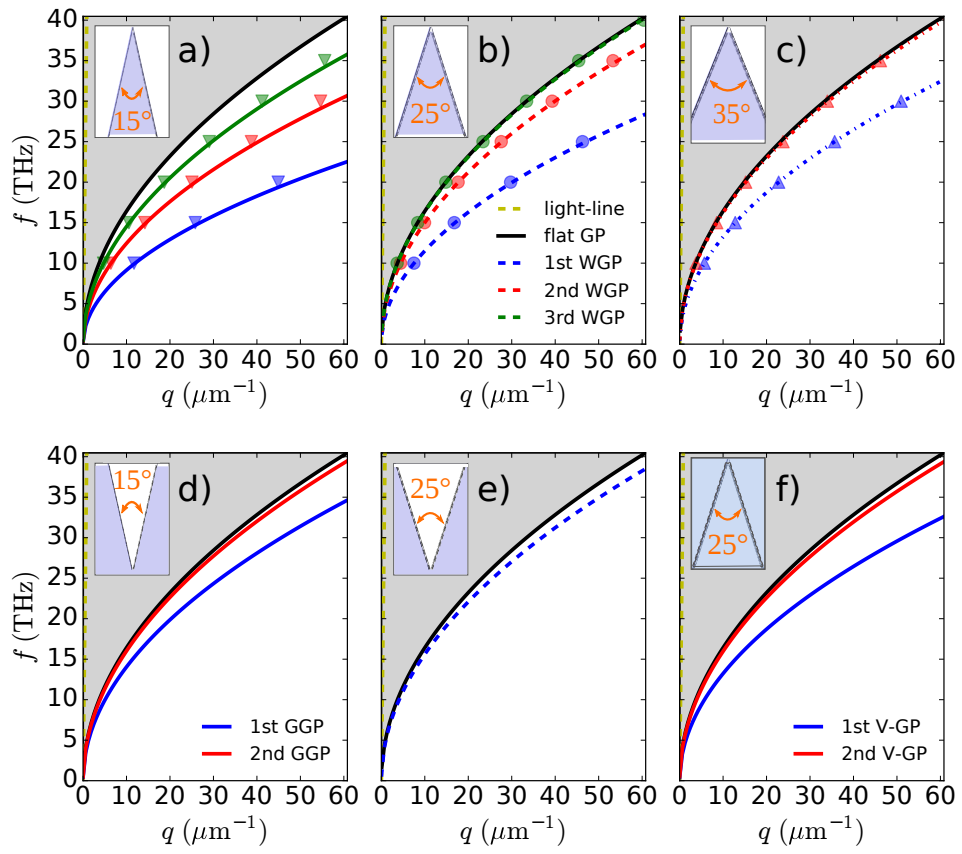

Figure 2: Spectrum of WGPs (upper row) and GGPs (first two bottom panels) sustained in different graphene-covered triangular configurations, with different opening angles, $2 \varphi$ (indicated in the insets), as given by Eq. ?? [we take $E_{F}=0.4 \mathrm{eV}$ ]. The solid black line represents the dispersion of GSPs in a flat interface and serves as reference. The colored $\nabla, \bigcirc$ and $\triangle$ data points figuring in the upper row correspond to the results for the WGP dispersion as obtained from full-wave numerical simulations (COMSOL's finite-element method). The insets' shading represents an insulator with $\varepsilon=4$, whereas the white regions denote a medium with $\varepsilon=1$ (e.g. air). The last panel shows the spectrum of GSPs guided along a V-shaped graphene embedded in a homogeneous medium with $\varepsilon=2.5$ (shaded in the inset as $\square$ ). 
modes attain increasingly larger wavevectors as we move from a groove, embedded and wedge configuration (in this order). This hints us that the ability to reach deep subwavelength regimes strongly depends on the $\varepsilon_{2} / \varepsilon_{1}$ ratio, for a fixed (acute) angle. Such prediction is confirmed by Fig. 3, in which we observe that the scaling factor appearing in Eq. (??) decreases monotonically with increasing $\varepsilon_{2} / \varepsilon_{1}$. In turn, this translates into higher effective indexes, $n_{\mathrm{eff}}=q / k_{0}$, for larger quotients $\varepsilon_{2} / \varepsilon_{1}$. As an example, we have obtained $n_{\text {eff }} \simeq 72$ for a frequency of $20 \mathrm{THz}$ in a configuration corresponding to panel in Fig. 2b. Even larger effective indexes can be obtained at higher frequencies (for instance, for the $\mathrm{CO}_{2}$ laser wavelength of $10.6 \mu \mathrm{m}$ this value climbs to $n_{\text {eff }} \simeq 100$, using the same parameters). Naturally, the higher amount of field localization promoted by the

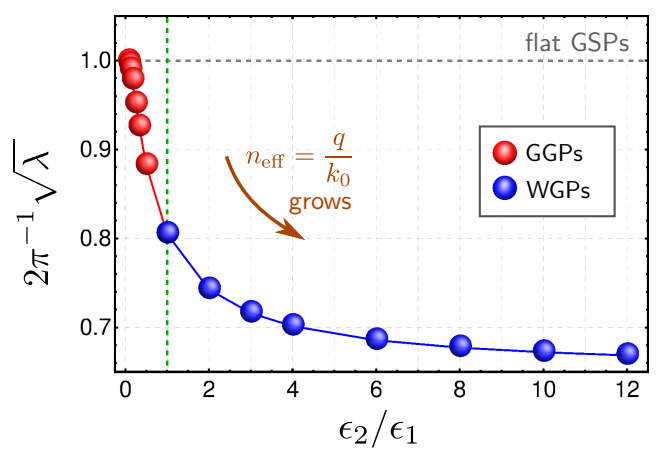

Figure 3: Dependence of the eigenvalues akin to the fundamental mode - in fact, of the proportionality factor, $2 \pi^{-1} \sqrt{\lambda}$, figuring in Eq. (??)—as a function of the ratio $\varepsilon_{2} / \varepsilon_{1}$. In computing the data in blue we have fixed $\varepsilon_{1}=1$ while varying $\varepsilon_{2}$, and vice-versa for the red data points. The vertical green dashed line indicates the point where $\varepsilon_{1}=\varepsilon_{2}=1$, whereas the horizontal gray dashed line sets the upper bound corresponding to the flat GSP dispersion. We further note that the value of $\lambda$ is also sensitive to the absolute value of the dielectric constant that is kept constant while the other varies.

WGP/GPP modes comes hand in hand with slightly larger propagation losses (cf. SI), a trait that is well-known and characteristic of plasmonics. Still, we have found that the number of plasmon oscillations within a propagation length ${ }^{454}$ remains unaltered when comparing between different mode orders or flat-GSPs (see SI for further details). Indeed, owing to the "universal scaling" epitomized by Eqs. (??) and (??), the ratio between loss and field confinement is independent of the mode order and is the same as for flat-GSPs.

Before concluding the analysis of Fig. 2, we highlight the capability of these guided GSPs 
modes to render extreme light-localization. This ability can be appreciated by considering the distance of their dispersion curves to the light-line (yellowish dashed-line near the vertical axis) and the large effective indexes that were obtained. This departure from the light-cone also justifies the high accuracy in the treatment of these modes within the electrostatic limit, as retardation effects are negligible and GSPs possess an essentially electrostatic character.

Potential and electric-field distributions. Furthermore, we remark that the solution of the eigenproblem in Eq. (??) also allows us to reconstruct the electrostatic potential within the graphene by feeding the obtained eigenvalues and eigenvectors back into the expansion for $\phi(r)$. From here, the $2 \mathrm{D}$ particle-density directly follows via $n(r)=\frac{\sigma(\omega)}{i e \omega}\left[\partial^{2} / \partial r^{2}-q^{2}\right] \phi(r)$ (see SI). Both these physical quantities, evaluated at the graphene's surface, that is $\theta=\varphi$ (at $\theta=-\varphi$ the distributions are antisymmetric), are shown in Fig. 4. Here, we consider WGPs with frequency $f=20 \mathrm{THz}$ guided along a $2 \varphi=25^{\circ}$ triangular wedge (which corresponds to the case illustrated in Fig. 2b).
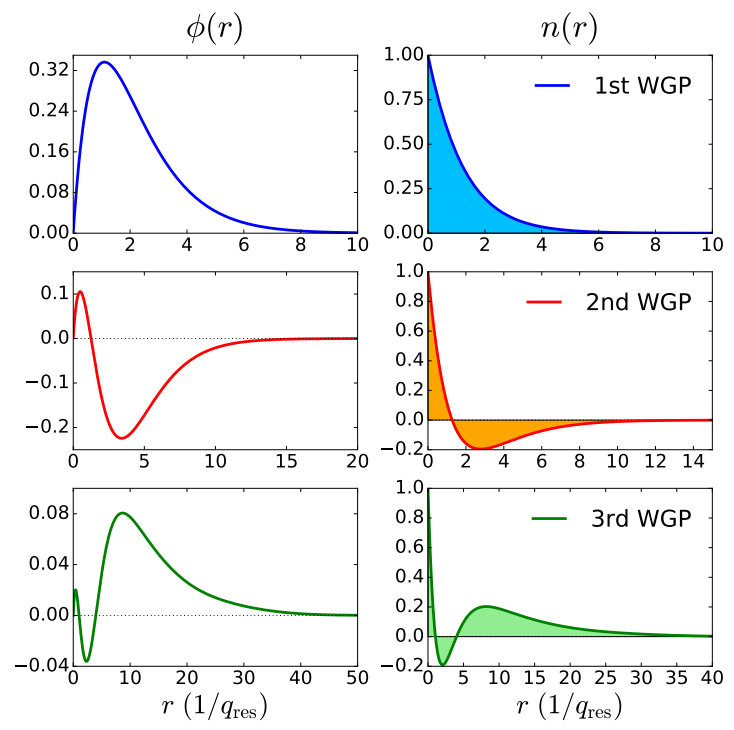

Figure 4: Radial distributions of the electrostatic potential, $\phi(r) \equiv \Phi(r, \varphi)$, and 2D particle density, $n(r)$, for the three lowest eigenmodes of a $25^{\circ}$ wedge covered with graphene (in a.u.), at a resonant frequency of $f=20 \mathrm{THz}$. The radial coordinate is plotted in units of the inverse wavevector akin to each mode [obtained by inverting Eq. (??) for a angular frequency of $\omega=2 \pi \times 20 \mathrm{THz}$ ]. The remaining parameters are the same as in Fig. $2 \mathrm{p}$ (i.e., $\varepsilon_{1}=1, \varepsilon_{2}=4$, and $E_{F}=0.4 \mathrm{eV}$ ).

Figure 4 indicates that the highest density of charge-carriers occurs at the apex of the wedge, 
irrespective of the mode order, although the density drops towards zero at increasingly larger distances from the origin for the higher-order modes. In addition, we note that $q_{3 \mathrm{rd}}^{-1}>q_{2 \mathrm{nd}}^{-1}>q_{1 \mathrm{st}}^{-1}$ for the same frequency, which makes the difference in the confinement even more dramatic. We emphasize that the number of nodes of both $\phi(r)$ and $n(r)$ is given by $m-1$, where $m=1,2, \ldots$ (for modes below the flat GSP dispersion curve) stands for the mode order. On the other hand, the value of the potential evaluated at the graphene layer is large near the vertex of the triangular cross-section, specially for the fundamental mode (where it is maximum). We acknowledge that, however, it is not located exactly at $r=0$. This is consequence of the infinitely sharp apex, whose corresponding Green's functions strongly oscillate at very small $r$, since they are not well-defined (but are bounded, i.e. do not diverge) in the $r \rightarrow 0$ limit. Conversely, for modes with higher energy, the potential tends to shift its weight farther from the apex of the V-structure as the mode order increases. Such behavior was already expected in the light of Fig. 2, since the WGP wavelength becomes smaller as the mode order decreases; as a consequence, the fundamental WGP mode exhibits the highest field-confinement, thereby being able to probe deeper into the V-wedge owing to its shorter wavelength when compared to the higher branches of the polaritonic spectrum. Again, we stress that although our model assumes infinitely deep triangular cross-sections, it can accurately describe finite-sized V-shapes as long as the height (depth) of the wedge (groove) is located at a distance somewhere along the "tail" of the quantities plotted in Fig. 4 (where they are essentially zero). In passing, we note that apart from the plasmon modes located at the apex of, say, a wedge ( $2 \varphi$ opening angle), the modes sustained at the corresponding "grooves", originating from the truncation of the structure-forming a $\pi / 2+\varphi$ angle - , can also be determined using the same guidelines as above, provided that the height of the wedge is large enough to prevent the hybridization of the modes. The same reasoning also holds for grooves.

We recall that once in possession of the potential evaluated at the graphene, one can build the potential in the entire coordinate space using Eq. (??). From here, the corresponding induced electric field follows directly by taking the gradient, i.e. $\mathbf{E}(\mathbf{r})=-\nabla \Phi(\mathbf{r})$. In what follows, we shall discuss only the spatial distributions of the potential and electric field within the plane transverse 
to the propagation direction, since its dependence along the $z$-axis is trivial due to the translational invariance of the system along this direction. The calculated 2D distributions (in the $x y$-plane) of the potential and concomitant electric field akin to WGPs in a representative $2 \varphi=25^{\circ}$ dielectric wedge are shown Fig. 5. Note that the electric field in cartesian coordinates can be fetched from its

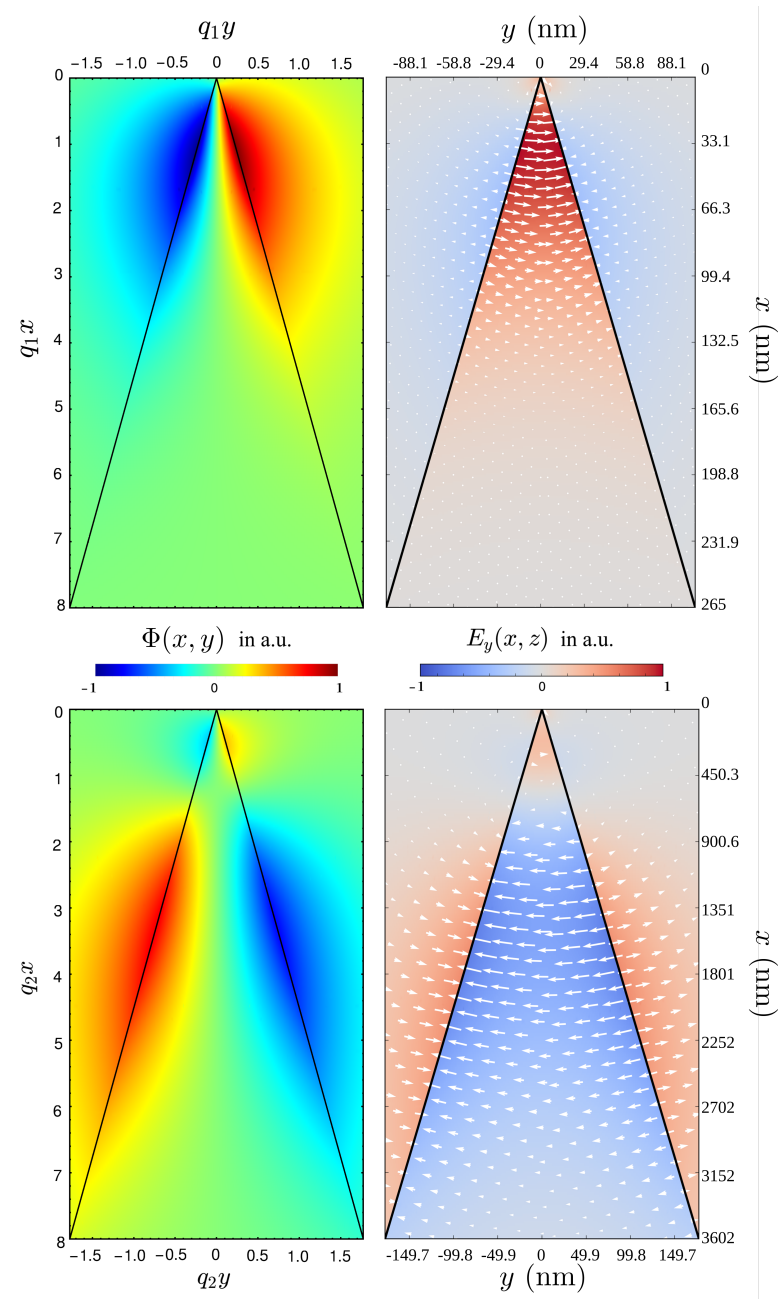

Figure 5: Two-dimensional potential, $\Phi(x, y)$, and electric-field distributions, $\mathbf{E}(x, y)=$ $-\nabla_{2 D} \Phi(x, y)$, ascribed to the fundamental (upper panels) and second order (lower panels) WGPs modes, at a resonant frequency of $f=20 \mathrm{THz}$. The parameters used in these computations match the ones in Fig. 4. The plotted region is the same in each pair of 2D plots, with axes of the panels on the left written in dimensionless units (normalized to the corresponding propagation constants) and the axes of the plots on the right are given in nanometers.

polar version by applying a rotation matrix, namely $\left(E_{x}, E_{y}\right)^{T}=R(\varphi)\left(E_{r}, E_{\theta}\right)^{T}$. The figure exhibits telling evidence of the remarkable confinement WGP are able to attain near the apex of the wedge (the results for the groove are qualitatively similar, albeit with slightly less localization for the same 
resonant frequency); this can be observed both from the induced potential or the electric-field. The intensity plot in the background of the vectorial representation of the $2 \mathrm{D} \mathbf{E}(x, y)$ refers to the value of the $y$-component of this quantity, which is dominant in most of the spatial region. Notice that since the potential has odd symmetry with relation to the $y=0$ plane, then the correspondent component of the electric field must be of even symmetry. It should also be highlighted that the vertical confinement (with respect to the apex edge) decreases rather rapidly as we move from the fundamental resonance to the higher order branches of the polaritonic spectrum. For the particular case depicted in the figure, the lowest energy mode essentially remains localized within the first 200 $\mathrm{nm}$, whereas for the second order mode that distance grows beyond $3.6 \mu \mathrm{m}$. Figure 5 also serves to support our previous claim that despite inherent simplifications in our theory, it may still be applied to realistic wedges (grooves) of finite height (depth). More specifically, the results portrayed in the figure accurately describe the 1st WGP in a 200nm-tall dielectric wedge, or, instead, both WGPs modes in a wedge with about $4 \mu \mathrm{m}$ in height.

The behavior of the spatial distributions displayed in Fig. 5 is qualitatively maintained throughout most of the dispersion curve (and similarly for different angles), the only important difference being the degree of confinement in the whereabouts of the apex edge. Therefore, an infinitely vast number of V-shaped geometric configurations, with different angles, heights or depths, may be engineered depending on the required level of localization and/or frequency region of interest. In this regard, one can use our model to effectively design and architecture a device which meets the pre-established requirements in terms of operating frequency range and dimensions.

\section{Concluding Remarks and Outlook}

In summary, we envision the exploitation of the folding of an otherwise planar graphene sheet—or any other SPP-supporting 2D material-into an (out-of-plane) triangular-like shape as a mean to achieve deep subwavelength waveguiding and light-localization using the plasmon modes guided along the apex of a V-shaped substrate. The fabrication of such devices is well within reach of 
current experimental state-of-the-art capabilities, as much of the technology has already been developed in the context of traditional 3D metal plasmonics. As an example, nanoimprint approaches

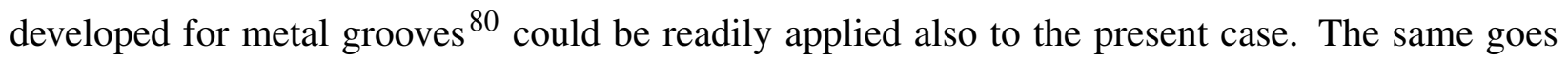
in what concerns potential mechanisms to excite these modes, since the same techniques used to excite CPPs could also be applied to excite WGP/GGP modes (e.g. end-fire coupling or excitation by fast electrons). $\frac{36}{1 n}$ Inis article, we have outlined a quasi-analytic method to theoretically describe graphene plasmons either guided along the ridge of a dielectric wedge or along the valley of a groove carved in an insulating substrate. The model accurately obtains the spectrum of the plasmonic eigenmodes supported by the aforementioned structures, as well as the potential and electric field distributions akin to those excitations. The computed modal distributions advocate the ability of these modes to achieve large field-enhancements and to deliver strong light-localization in the neighborhood of the triangular edge. Interestingly, we have found that the dispersion of each particular WGP/GGP mode obeys a universal scaling law, in the sense that the functional dependence of the flat-GSP spectrum is maintained up to a multiplication constant that depends solely on the particular geometric configuration of the system (i.e., the angle $\varphi$ for a given $\varepsilon_{1}$ and $\varepsilon_{2}$ ). The results of our calculations were verified by performing rigorous electrodynamic simulations based on the FEM, to which a very good agreement was observed. We note, however, that the quasianalytical model presented above not only provides more physical insight when gauged against numerical simulations, but is also far less computationally demanding and less time-consuming that the latter, since all the eigenmodes for a given structure can be determined at once from a single computation. Moreover, we have showed that WGPs and GPPs may be suitable candidates for versatile platforms (specially when taking advantage of the gate-tunability of graphene plasmons) to effectively route highly confined EM radiation. In this context, subsequent theoretical investigations of wedges/grooves with more realistic, rounded edges should constitute the basis of future work. The rounding of the apex leads to a slight decrease in the effective index of the plasmon modes, as shown by our finite-element simulations (cf. SI). Nevertheless, we expect our results to be qualitatively robust with respect to rounding. Furthermore, it was demonstrated that even 
for infinitely sharp metallic apexes, non-local effects prevent the emergence of singularities at the tip. ${ }^{81}$ The case of a 2D layer deposited on a V-shaped substrate with a frequency-dependent dielectric function, e.g. a metal or a polar medium, can also be considered using the theory developed here. In addition, the effects of strain owing to the folding of the 2D sheet around the apex remain largely unexplored in what concerns their implications in plasmonic excitations [for instance, in electronic transport it is well-known that strain introduces a scattering potential for the 2D Dirac fermions (via a gauge field) which can lead to the modification of the bandstructure]. ${ }^{82[83}$ We thus expect that this work will fuel future experimental realizations of WGPs/GGPs, as we believe that such modes may hold interesting implications for future all-photonic circuitries at the nanoscale.

\section{Acknowledgement}

PADG, SX and NAM acknowledge financial support from the Danish National Research Foundation through the sponsoring of the Center for Nanostructured Graphene (CNG), Project DNRF103. NMRP acknowledges funding from the European Commission within of the project "GrapheneDriven Revolutions in ICT and Beyond" (Ref. No. 696656), the Portuguese Foundation for Science and Technology (FCT) in the framework of the Strategic Financing UID/FIS/04650/2013, and the hospitality of the MackGraphe Center, Mackenzie Presbyterian University.

\section{Supporting Information Available}

Supporting Information Available: In the supporting information we describe the derivation of the equations given in the main article with utmost detail, while also outlining the calculation of the Green's function akin to a V-shaped interface between two insulators. Finally, we give further information about propagation losses, and critically analyze the dependence of the concurrently conducted finite element method (FEM) simulations on the radius of the curvature at the triangular apex, since the numerical simulations - contrarily to the analytics-cannot rigorously deal with the sharp tip of the V-structure.

This material is available free of charge via the Internet at http://pubs.acs.org/. 


\section{References}

(1) Saleh, B. E. A.; Teich, M. C. Fundamentals of Photonics, 2nd ed.; Wiley, 2012.

(2) Atwater, H. A. The Promise of Plasmonics. Sci. Am. 2007, 296, 56-62.

(3) Maradudin, A. A.; Barnes, W. L.; Sambles, J. R. Modern Plasmonics; Elsevier, 2014.

(4) Maier, S. A. Plasmonics: Fundamentals and Applications; Springer: New York, 2007.

(5) Barnes, W. L.; Dereux, A.; Ebbesen, T. W. Surface plasmon subwavelength optics. Nature 2003, 424, 824-830.

(6) Armstrong, S. Plasmonics: Diffraction-free surface waves. Nature Photon. 2012, 6, 720.

(7) Barnes, W. L. Surface plasmon-polariton length scales: a route to sub-wavelength optics. $J$. Opt. A: Pure Appl. Opt. 2006, 8, S87.

(8) Gramotnev, D. K.; Bozhevolnyi, S. I. Plasmonics beyond the diffraction limit. Nature Photon. 2010, 4, 83-91.

(9) Ozbay, E. Plasmonics: Merging Photonics and Electronics at Nanoscale Dimensions. Science 2006, 311, 189-193.

(10) Bozhevolnyi, S. I.; Volkov, V. S.; Devaux, E.; Laluet, J.-Y.; Ebbesen, T. W. Channel plasmon subwavelength waveguide components including interferometers and ring resonators. Nature 2006, 440, 508-511.

(11) Han, Z.; Bozhevolnyi, S. I. Radiation guiding with surface plasmon polaritons. Rep. Prog. Phys. 2013, 76, 016402.

(12) Bozhevolnyi, S. I. Plasmonic Nanoguides and Circuits; Pan Stanford: Singapore, 2008.

(13) Bergman, D. J.; Stockman, M. I. Surface Plasmon Amplification by Stimulated Emission of Radiation: Quantum Generation of Coherent Surface Plasmons in Nanosystems. Phys. Rev. Lett. 2003, 90, 027402. 
(14) Berini, P.; Leon, I. D. Surface plasmon-polariton amplifiers and lasers. Nature Photon. 2012, $6,16-24$.

(15) Ma, R.-M.; Oulton, R. F.; Sorger, V. J.; Zhang, X. Plasmon lasers: coherent light source at molecular scales. Laser Photon. Rev. 2013, 7, 1-21.

(16) Haffner, C. et al. All-plasmonic Mach-Zehnder modulator enabling optical high-speed communication at the microscale. Nature Photon. 2015, 9, 525.

(17) Chang, D. E.; Sørensen, A. S.; Hemmer, P. R.; Lukin, M. D. Quantum Optics with Surface Plasmons. Phys. Rev. Lett. 2006, 97, 053002.

(18) Heeres, R. W.; Kouwenhoven, L. P.; Zwiller, V. Quantum interference in plasmonic circuits. Nature Nanotechnol. 2013, 8, 719-722.

(19) Fakonas, J. S.; Lee, H.; Kelaita, Y. A.; Atwater, H. A. Two-plasmon quantum interference. Nature Photon. 2014, 8, 317-320.

(20) Tame, M. S.; McEnery, K. R.; Ozdemir, S. K.; Lee, J.; Maier, S. A.; Kim, M. S. Quantum plasmonics. Nature Phys. 2013, 9, 329-340.

(21) Bermudez-Urena, E.; Gonzalez-Ballestero, C.; Geiselmann, M.; Marty, R.; Radko, I. P.; Holmgaard, T.; Alaverdyan, Y.; Moreno, E.; García-Vidal, F. J.; Bozhevolnyi, S. I.; Quidant, R. Coupling of individual quantum emitters to channel plasmons. Nature Commun. 2015, 6, 7883 .

(22) Aćimović, S. S.; Ortega, M. A.; Sanz, V.; Berthelot, J.; Garcia-Cordero, J. L.; Renger, J.; Maerkl, S. J.; Kreuzer, M. P.; Quidant, R. LSPR Chip for Parallel, Rapid, and Sensitive Detection of Cancer Markers in Serum. Nano Lett. 2014, 14, 2636-2641.

(23) Haes, A. J.; Haynes, C. L.; McFarland, A. D.; Schatz, G. C.; Duyne, R. P. V.; Zou, S. Plasmonic Materials for Surface-Enhanced Sensing and Spectroscopy. MRS Bulletin 2005, 30, $368-375$. 
(24) Brolo, A. G. Plasmonics for future biosensors. Nature Photon. 2012, 6, 709-713.

(25) Long, Y.-T.; Jing, C. Localized Surface Plasmon Resonance Based Nanobiosensors; Springer, 2014.

(26) Rodrigo, D.; Limaj, O.; DavideJanner,; Etezadi, D.; García de Abajo, F. J.; Pruneri, V.; Altug, H. Mid-infrared plasmonic biosensing with graphene. Science 2015, 349, 165-168.

(27) Fang, N.; Lee, H.; Sun, C.; Zhang, X. Sub-Diffraction-Limited Optical Imaging with a Silver Superlens. Science 2005, 308, 534-537.

(28) Liu, N.; Hentschel, M.; Weiss, T.; Alivisatos, A. P.; Giessen, H. Three-Dimensional Plasmon Rulers. Science 2011, 332, 1407-1410.

(29) Vo-Dinh, T.; Fales, A. M.; Griffin, G. D.; Khoury, C. G.; Liu, Y.; Ngo, H.; Norton, S. J.; Register, J. K.; Wang, H.-N.; Yuan, H. Plasmonic nanoprobes: from chemical sensing to medical diagnostics and therapy. Nanoscale 2013, 5, 10127-10140.

(30) Zheng, Y. B.; Kiraly, B.; Weiss, P. S.; Huang, T. J. Molecular plasmonics for biology and nanomedicine. Nanomedicine 2012, 7, $751-770$.

(31) Bardhan, R.; Lal, S.; Joshi, A.; Halas, N. J. Theranostic Nanoshells: From Probe Design to Imaging and Treatment of Cancer. Accounts of Chemical Research 2011, 44, 936-946.

(32) Fang, Y.; Sun, M. Nanoplasmonic waveguides: towards applications in integrated nanophotonic circuits. Light Sci. Appl. 2015, 4, e294.

(33) Krenn, J. R. Nanoparticle waveguides: Watching energy transfer. Nature Mater. 2003, 2 , 210-211.

(34) Maier, S. A.; Kik, P. G.; Atwater, H. A.; Meltzer, S.; Harel, E.; Koel, B. E.; Requicha, A. A. G. Local detection of electromagnetic energy transport below the diffraction limit in metal nanoparticle plasmon waveguides. Nature Mater. 2003, 2, 229-232. 
(35) Alù, A.; Belov, P. A.; Engheta, N. Coupling and guided propagation along parallel chains of plasmonic nanoparticles. New J. Phys. 2011, 13, 033026.

(36) Smith, C. L. C.; Stenger, N.; Kristensen, A.; Mortensen, N. A.; Bozhevolnyi, S. I. Gap and channeled plasmons in tapered grooves: a review. Nanoscale 2015, 7, 9355-9386.

(37) Gramotnev, D. K.; Bozhevolnyi, S. I. Nanofocusing of electromagnetic radiation. Nature Photon. 2014, 9, 13-22.

(38) Raza, S.; Stenger, N.; Pors, A.; Holmgaard, T.; Kadkhodazadeh, S.; Wagner, J. B.; Pedersen, K.; Wubs, M.; Bozhevolnyi, S. I.; Mortensen, N. A. Extremely confined gap surfaceplasmon modes excited by electrons. Nature Commun. 2014, 5, 4125.

(39) Pile, D. F. P.; Gramotnev, D. K. Channel plasmon-polariton in a triangular groove on a metal surface. Opt. Lett. 2004, 29, 1069-1071.

(40) Moreno, E.; García-Vidal, F. J.; Rodrigo, S. G.; Martin-Moreno, L.; Bozhevolnyi, S. I. Channel plasmon-polaritons: modal shape, dispersion, and losses. Opt. Lett. 2006, 31, 3447-3449.

(41) Li, X.; Jiang, T.; Shen, L.; Deng, X. Subwavelength guiding of channel plasmon polaritons by textured metallic grooves at telecom wavelengths. Appl. Phys. Lett. 2013, 102, 031606.

(42) Volkov, V. S.; Bozhevolnyi, S. I.; Devaux, E.; Ebbesen, T. W. Compact gradual bends for channel plasmon polaritons. Opt. Express 2006, 14, 4494-4503.

(43) Dobrzynski, L; Maradudin, A. A. Electrostatic Edge Modes in a Dielectric Wedge. Phys. Rev. B 1972, 6, 3810-3815.

(44) Boardman, A. D.; Aers, G. C.; Teshima, R. Retarded edge modes of a parabolic wedge. Phys. Rev. B 1981, 24, 5703-5712.

(45) Boardman, A. D.; Garcia-Molina, R.; Gras-Marti, A.; Louis, E. Electrostatic edge modes of a hyperbolic dielectric wedge: Analytical solution. Phys. Rev. B 1985, 32, 6045-6047. 
(46) Novikov, I. V.; Maradudin, A. A. Channel polaritons. Phys. Rev. B 2002, 66, 035403.

(47) Lu, J. Q.; Maradudin, A. A. Channel plasmons. Phys. Rev. B 1990, 42, 11159-11165.

(48) Volkov, V. S.; Bozhevolnyi, S. I.; Rodrigo, S. G.; Martín-Moreno, L.; García-Vidal, F. J.; Devaux, E.; Ebbesen, T. W. Nanofocusing with Channel Plasmon Polaritons. Nano Lett. 2009, 9, 1278-1282.

(49) Bozhevolnyi, S. I.; Nerkararyan, K. V. Channel plasmon polaritons guided by graded gaps: closed-form solutions. Opt. Express 2009, 17, 10327-10334.

(50) Bozhevolnyi, S. I.; Nerkararyan, K. V. Analytic description of channel plasmon polaritons. Opt. Lett. 2009, 34, 2039-2041.

(51) Luo, Y.; Pendry, J. B.; Aubry, A. Surface Plasmons and Singularities. Nano Letters 2010, 10, 4186-4191.

(52) Geim, A. K. Graphene: Status and Prospects. Science 2009, 324, 1530-1534.

(53) Castro Neto, A. H.; Guinea, F.; Peres, N. M. R.; Novoselov, K. S.; Geim, A. K. The electronic properties of graphene. Rev. Mod. Phys. 2009, 81 .

(54) Gonçalves, P. A. D.; Peres, N. M. R. An Introduction to Graphene Plasmonics; World Scientific: Singapore, 2016.

(55) García de Abajo, F. J. Graphene Plasmonics: Challenges and Opportunities. ACS Photonics 2014, $1,135-152$.

(56) Xiao, S.; Zhu, X.; Li, B.-H.; Mortensen, N. A. Graphene-plasmon polaritons: From fundamental properties to potential applications. Front. Phys. 2016, 11, 117801.

(57) Bludov, Y. V.; Ferreira, A.; Peres, N. M. R.; Vasilevskiy, M. I. A Primer on Surface PlasmonPolaritons in Graphene. Int. J. Mod. Phys. B 2013, 27, 1341001. 
(58) Low, T.; Avouris, P. Graphene Plasmonics for Terahertz to Mid-Infrared Applications. ACS Nano 2014, 8, 1086-1101.

(59) Koppens, F. H. L.; Chang, D. E.; García de Abajo, F. J. Graphene Plasmonics: A Platform for Strong Light-Matter Interactions. Nano Lett. 2011, 11, 3370-3377.

(60) Ju, L.; Geng, B.; Horng, J.; Girit, C.; Martin, M.; Hao, Z.; Bechtel, H. A.; Liang, X.; Zettl, A.; Shen, Y. R.; Wang, F. Graphene plasmonics for tunable terahertz metamaterials. Nature Nanotechnol. 2011, 6, 630-634.

(61) Yan, H.; Low, T.; Zhu, W.; Wu, Y.; Freitag, M.; Li, X.; Guinea, F.; Avouris, P.; Xia, F. Damping pathways of mid-infrared plasmons in graphene nanostructures. Nature Photon. 2013, 7, 394-399.

(62) Luxmoore, I. J.; Gan, C. H.; Liu, P. Q.; Valmorra, F.; Li, P.; Faist, J.; Nash, G. R. Strong coupling in the far-infrared between graphene plasmons and the surface optical phonons of silicon dioxide. ACS Photonics 2014, 1, 1151.

(63) Hu, H.; Yang, X.; Zhai, F.; Hu, D.; Liu, R.; Liu, K.; Sun, Z.; Dai, Q. Far-field nanoscale infrared spectroscopy of vibrational fingerprints of molecules with graphene plasmons. Nature Commun. 2016, 7, 1151.

(64) Yan, H.; Xia, F.; Li, Z.; Avouris, P. Plasmonics of coupled graphene micro-structures. New J. Phys. 2012, 14, 125001.

(65) Fang, Z.; Thongrattanasiri, S.; Schlather, A.; Liu, Z.; Ma, L.; Wang, Y.; Ajayan, P. M.; Nordlander, P.; Halas, N. J.; García de Abajo, F. J. Gated Tunability and Hybridization of Localized Plasmons in Nanostructured Graphene. ACS Nano 2013, 7, 2388-2395.

(66) Fang, Z.; Wang, Y.; Schlather, A. E.; Liu, Z.; Ajayan, P. M.; García de Abajo, F. J.; Nordlander, P.; Zhu, X.; Halas, N. J. Active Tunable Absorption Enhancement with Graphene Nanodisk Arrays. Nano Lett. 2014, 14, 299-304. 
(67) Zhu, X.; Wang, W.; Yan, W.; Larsen, M. B.; Bøggild, P.; Pedersen, T. G.; Xiao, S.; Zi, J.; Mortensen, N. A. Plasmon-Phonon Coupling in Large-Area Graphene Dot and Antidot Arrays Fabricated by Nanosphere Lithography. Nano Lett. 2014, 14, 2907-2913.

(68) Wang, Z.; Li, T.; Almdal, K.; Mortensen, N. A.; Xiao, S.; Ndoni, S. Pushing graphene plasmon polaritons to the near-infrared window by block copolymer nanolithography. arXiv:1606.02471 [physics.optics] 2016,

(69) Liu, P. Q.; Valmorra, F.; Maissen, C.; Faist, J. Electrically tunable graphene anti-dot array terahertz plasmonic crystals exhibiting multi-band resonances. Optica 2015, 2, 135-140.

(70) Yeung, K. Y. M.; Chee, J.; Yoon, H.; Song, Y.; Kong, J.; Ham, D. Far-Infrared Graphene Plasmonic Crystals for Plasmonic Band Engineering. Nano Lett. 2014, 14, 2479-2484.

(71) Liu, P.; Zhang, X.; Ma, Z.; Cai, W.; Wang, L.; Xu, J. Surface plasmon modes in graphene wedge and groove waveguides. Opt. Express 2013, 21, 32432-32440.

(72) Kim, K.; Lee, Z.; Malone, B. D.; Chan, K. T.; Alemán, B.; Regan, W.; Gannett, W.; Crommie, M. F.; Cohen, M. L.; Zettl, A. Multiply folded graphene. Phys. Rev. B 2011, 83, 245433.

(73) Pakhnevich, A. A.; Golod, S. V.; Prinz, V. Y. Surface melting of copper during graphene growth by chemical vapour deposition. J. Phys. D: Appl. Phys. 2015, 48, 435303.

(74) Song, Q.; An, M.; Chen, X.; Peng, Z.; Zang, J.; Yang, N. Adjustable thermal resistor by reversibly folding a graphene sheet. Nanoscale $\mathbf{2 0 1 6}$,

(75) Zhu, W.; Low, T.; Perebeinos, V.; Bol, A. A.; Zhu, Y.; Yan, H.; Tersoff, J.; Avouris, P. Structure and Electronic Transport in Graphene Wrinkles. Nano Lett. 2012, 12, 3431-3436.

(76) Wu, J.-W.; Hawrylak, P.; Eliasson, G.; Quinn, J.; Fetter, A. Magnetoplasma surface waves on the lateral surface of a semiconductor superlattice. Sol. State Commun. 1986, 58, 795-798.

(77) Wang, W.; Apell, P.; Kinaret, J. Edge plasmons in graphene nanostructures. Phys. Rev. B 2011, 84, 085423. 
(78) Abramowitz, M.; Stegun, I. A. Handbook of Mathematical Functions: with Formulas, Graphs, and Mathematical Tables; Dover: New York, 1965.

(79) Harrison, P. Quantum Wells, Wires and Dots, 3rd ed.; Wiley, 2010.

(80) Nielsen, R. B.; Fernandez-Cuesta, I.; Boltasseva, A.; Volkov, V. S.; Bozhevolnyi, S. I.; Klukowska, A.; Kristensen, A. Channel plasmon polariton propagation in nanoimprinted Vgroove waveguides. Opt. Lett. 2008, 33, 2800-2802.

(81) Toscano, G.; Raza, S.; Yan, W.; Jeppesen, C.; Xiao, S.; Wubs, M.; Jauho, A.-P.; Bozhevolnyi, S. I.; Mortensen, N. A. Nonlocal response in plasmonic waveguiding with extreme light confinement. Nanophotonics 2013, 2, 161-166.

(82) de Juan, F.; Mañes, J. L.; Vozmediano, M. A. H. Gauge fields from strain in graphene. Phys. Rev. B 2013, 87, 165131.

(83) Amorim, B.; Cortijo, A.; de Juan, F.; Grushin, A.; Guinea, F.; Gutiérrez-Rubio, A.; Ochoa, H.; Parente, V.; Roldán, R.; San-Jose, P.; Schiefele, J.; Sturla, M.; Vozmediano, M. Novel effects of strains in graphene and other two dimensional materials. Physics Reports 2016, 617, 1 - 54, Novel effects of strains in graphene and other two dimensional materials. 
Graphical TOC Entry

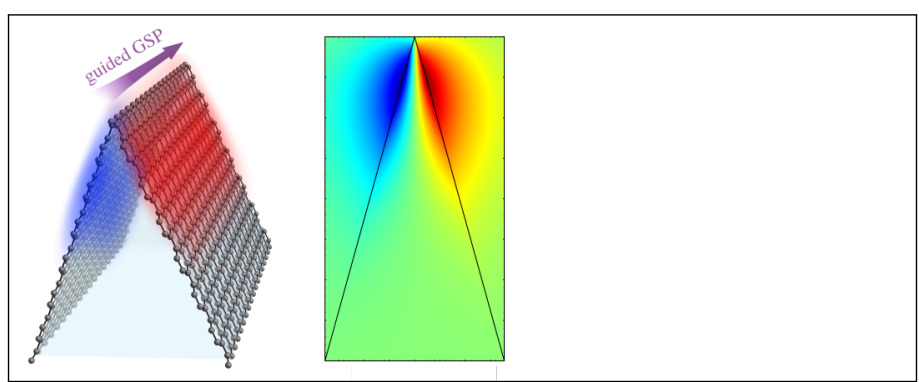

